\author{
B. Meylan ${ }^{\mathrm{a}}$, T. Le-Quang ${ }^{\mathrm{a}}$, M.P. Olbinado ${ }^{\mathrm{b}}$, A. Rack ${ }^{\mathrm{b}}$, S.A. Shevchik ${ }^{\mathrm{a}}$, K. Wasmer ${ }^{\mathrm{a}}$ \\ ${ }^{a}$ Laboratory for Advanced Materials Processing (LAMP), Swiss Federal Laboratories for Materials Science and Technology \\ (Empa), Thun, Switzerland
}

${ }^{\mathrm{b}}$ ESRF - The European Synchrotron, Grenoble, France

\title{
Re-solidification dynamics and microstructural analysis of laser welded aluminium
}

\author{
Paper presented at the Symposium "Tomographic and Radiographic Imaging with Synchrotron \\ X-rays and Neutrons" of the MSE 2018, 26-28 September 2018, Darmstadt, Germany
}

In this work, we investigated the re-solidification dynamics and microstructure of aluminium AA5005 welded by fibre laser. The re-solidification process was visualized by highspeed X-ray imaging at the European Synchrotron Radiation Facility (ESRF, Grenoble, France). The final microstructure was studied by electron backscatter diffraction. The experiments were performed in both conduction and keyhole weld regimes. The results show that fine and equiaxed structure is obtained for conduction welding due to very high cooling rate. Keyhole welding also shows similar structure at the beginning of re-solidification, followed by a columnar microstructure.

Keywords: Laser welding; X-ray imaging; Microstructure

\section{Introduction}

Laser technology has been has been involved in a large number of industrial applications, e.g. in the automotive, electronics and medical sectors. Among them, laser welding is one of the most popular. This process involves rapid melting of materials by an intense laser beam, followed by fast re-solidification at the end of the irradiation [1]. There are two regimes of welding: conduction and keyhole welding. The former involves shallow melting of the sample's surface at low absorbed laser intensity and the melt pool expands solely by heat conduction. On the other hand, keyhole welding takes place at high laser intensity that is sufficient to cause intensive evaporation of the molten material. The recoil pressure pushes the liquid aside, allowing the laser beam to propagate further into the sample. This regime is characterized by high aspect ratio of the melt pool [1].

Obviously, laser welding is a highly dynamic process making it difficult to guarantee high reproducibility of weld quality [1]. Therefore, there is a growing demand for in-situ and real-time quality monitoring of this process. It has two aspects: the first one is on detecting the formation of defects such as porosity and cracks; the second aspect is on monitoring the microstructure of the welded zone $[1,2]$. Despite extensive studies on the underlying physics, the realization of such a system remains a challenge due to the involvement of numerous sub-millisecond events and the complexity of the laser-matter interaction [3-6]. For the former task, the present authors successfully demonstrated a promising approach based on the combination of acoustic emission (AE) with artificial intelligence in previous studies [3, 4]. Stateof-the-art high speed X-ray imaging instruments at the European Synchrotron Radiation Facility (ESRF) were used to elucidate the correlation between the $\mathrm{AE}$ signals and the transient events. The present study is a continuation of the work and will focus on the influence of the process parameters on the microstructure of the welded zone.

\section{Experiments}

The laser welding experiments were made with a single mode fibre laser source StarFiber 150P (Coherent, Switzerland) with a $1070 \mathrm{~nm}$ wavelength. The laser system was operated in pulse mode. The emitted Gaussian beam was transmitted through a $12 \mu \mathrm{m}$ diameter single - mode optical fiber to a laser head. The laser beam was guided by the optical system within the laser head and then focused on the sample surface by a focusing lens with a focal length of $170 \mathrm{~mm}$. The diameter of the laser spot at the focus was approximately $30 \mu \mathrm{m}$ at $1 / e^{2}$ of the beam's maximum intensity. This small diameter allowed keyhole formation even at low laser power.

The high-speed X-ray imaging was carried out at beamline ID19 of the ESRF using hard synchrotron radiation. $\mathrm{X}$-ray phase contrast imaging by free-space propagation was utilised to enhance the contrast between material interfaces. The beam mean energy was $26.3 \mathrm{keV}$. The recording frame rate during the experiments was fixed at $28762 \mathrm{fps}$. The effective pixel size of the X-ray image detector was $11 \mu \mathrm{m}$. The setup is depicted in Fig. 1 and detailed information can be found elsewhere [3].

In our experiments, aluminium AA5005 was chosen for the weld samples due to its high X-ray transmissivity. The aluminium samples manufactured for welding had a dimension of $2 \times 20 \times 50 \mathrm{~mm}^{3}$. The samples were placed to have a thickness of $2 \mathrm{~mm}$ along the X-ray path, allowing a transmission rate of approximately $54 \%$. The samples were firmly fixed in an aluminium sample holder which was placed on an $X Y$ table (Zaber Technology Inc., USA). Laser parameters such as pulse duration average laser power were varied in wide ranges to provoke different welding regimes. In particular, pulse duration in the range $10-12 \mathrm{~ms}$ was 
used. Keyhole welding was achieved with laser powers in the range 750-1200 W while lower laser powers correspond to conduction welding.

After the experiments, the samples were prepared for metallographic examination. They were cut and polished through the middle of the welds. After polishing, the samples were etched using Weck's reagent $(100 \mathrm{~mL}$ water with $4 \mathrm{~g} \mathrm{KMnO} 4$ and $1 \mathrm{~g} \mathrm{NaOH}$ ) for 30s and observed under an optical microscope. Then, the samples were polished again to perform EBSD (electron back scatter diffraction) measurements of the welds using an EBSD camera (EDAX Inc., USA) mounted on a Hitachi S4800 SEM.

\section{Results and discussion}

X-ray radiographic images of the melt pool during a typical keyhole experiments are displayed in Fig. 2. The pulse power and pulse duration were $1000 \mathrm{~W}$ and $10 \mathrm{~ms}$, respectively. At the beginning of the experiment $(t=0.3 \mathrm{~ms})$, only a small melt pool could be observed (blue arrow). Its geometry resembles the conduction welding regime. The occurrence of this regime is due to the high reflectivity of aluminium in solid phase which results in low absorbed radiation energy and, hence, rather low surface temperature [7]. As the irradiation continues, the sample temperature rises resulting in a decrease in surface reflectivity and so an increase in absorbed energy. This is particularly the case when the melting temperature is reached. At a sufficiently high tempera-

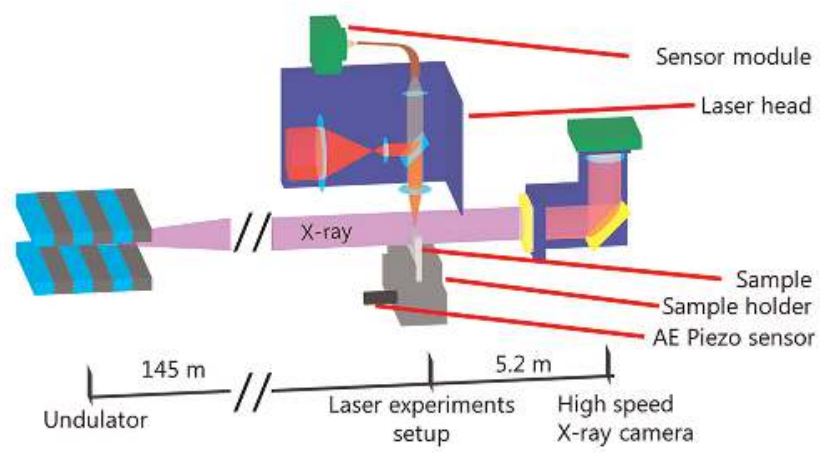

Fig. 1. Diagram of the experimental setup used in the present work. ture, intense evaporation of the molten material occurs and the keyhole channel is formed by the recoil pressure [8]. In this example, the keyhole becomes visible after $0.5 \mathrm{~ms}$ (red arrow). The keyhole can be easily distinguished in the $\mathrm{X}$ ray images due to its very low density as compared to the liquid and solid phases, leading to higher X-ray transmission. The appearance of the keyhole allows a deeper penetration of the laser beam and it significantly enhances the laser absorption by multiple reflections inside the channel [9]. Consequently, it causes a rapid increase in depth of the melt pool (see images at 1,2 and $3 \mathrm{~ms}$ ). As the keyhole grows in depth, its instability rises due to an augmentation of surface tension and, at the same time, a decrease in the recoil pressure [8]. This condition leads to fluctuations of the melt pool and undesirable events such as pore formation and spatter (see $t=4-10 \mathrm{~ms}$ ). Toward the end of the experiment, the keyhole forms a bulb at the bottom and a narrow opening at the top ( $t=10 \mathrm{~ms}$ ). This geometry is prejudicial to the weld quality, as it results in the formation of a pore (green arrow, $t=10.1 \mathrm{~ms}$ ) once the laser power is stopped and the keyhole collapses (see 10.1 s and after). The melt pool takes just less than $4 \mathrm{~ms}$ to completely solidify. After the solidification is complete, cracks are present in the recently solidified area (pink arrow, $t=14 \mathrm{~ms}$ ).

Figure 3 shows the evolution of the melt pool during a single pulse in the conduction regime. This pulse comes from a series of pulses with ramping power, to go from no melting $(100 \mathrm{~W})$ to keyhole $(1000 \mathrm{~W})$ and back to no melting. Each ramping period lasted 20 pulses and the laser power was fixed at $1000 \mathrm{~W}$ for 10 pulses. The series of pulses was realised with the aluminium sample moving from right to left, with respect to the view of the X-ray images, at $1.5 \mathrm{~mm} \cdot \mathrm{s}^{-1}$ and with a frequency of $10 \mathrm{~Hz}$. The previous pulse is on the left hand side of the actual pulse and some remaining porosity can be seen just below the surface (see pink arrow, $t=0 \mathrm{~ms}$ ). The pulse duration is $10 \mathrm{~ms}$ at a power of approximately $600 \mathrm{~W}$ situated after the region of high power when the power was on the decreasing ramp. The melt pool (see blue arrow, $t=4 \mathrm{~ms}$ ) is fully formed after around $4 \mathrm{~ms}$ and the size does not vary much till the end of the pulse at $10 \mathrm{~ms}$. After the end of the pulse, the size of the melt pool decreases rapidly and after $0.6 \mathrm{~ms}$, no more trace of liquid is present. The melt pools in conduction re-

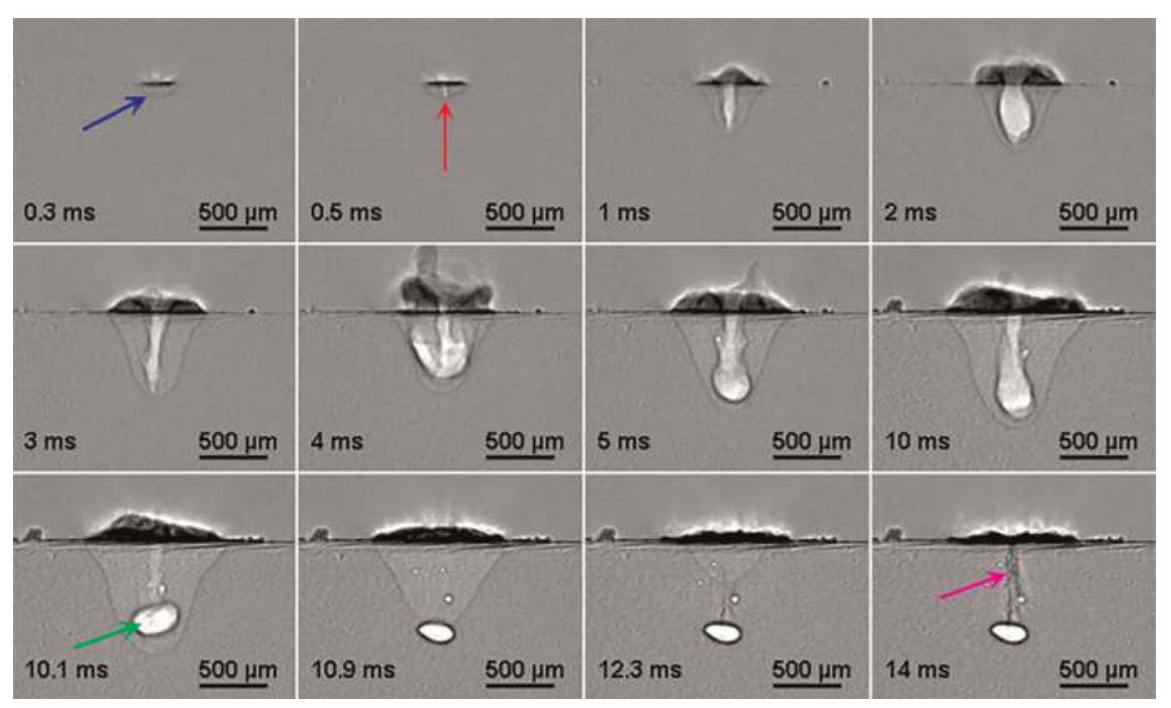

Fig. 2. X-ray radiograph of a single $10 \mathrm{~ms}$ pulse of $1000 \mathrm{~W}$ in the keyhole regime. Blue arrow shows the beginning of the melt pool; the red arrow shows the start of the keyhole; green arrow illustrates the pore formation and pink arrow points to the resulting cracks after solidification. 
gimes are small with a depth less than half their diameters. For our conditions, this corresponds to maximum depths of $100 \mu \mathrm{m}$ which correspond to only $9-10$ pixels on our Xray images. The reason is that the setup was designed predominantly to observe the keyhole regime. Despite this limitation, the X-ray images can still be used to observe the melt pool duration and to approximate its size.

To get a better measurement of the melt pool size, ex-situ destructive observations were performed and an example is shown in Fig. 4 for a series of pulses. Figure 4 a presents a cross-section of the region with a series of pulses with decreasing power. The pulse shown in the X-ray images in Fig. 3 corresponds to the fourth pulse from the left hand side of the image (see blue arrow). In Fig. 4a, each individual melt pool is revealed by etching the sample cross-section. In this figure, the melt pools appear in colour whereas the bulk microstructure has a uniform light grey. The micrograph (Fig. 4a) reveals as well small porosity (red arrows) that was too small to detect with the setup used for X-ray imag- ing. The micrograph also shows that the melt pool partially covers the previous laser pulse and is also partially covered by the next one. The covering percentage is not constant and depends of the laser power and varies from $50 \%$ for high power down to $20 \%$ for the lowest power.

EBSD analysis was performed on one of the melt pools shown in Fig. 4a, and the corresponding inverse pole figure map is presented in Fig. 4b. However, the exact melt pool is difficult to find on the optical picture of Fig. 4a as the sample was re-polished between the measurements and the landmarks (e.g. pores) are not found at the same place. Based on the size of the inspected melt pool, it corresponds to the $5^{\text {th }}$ or $6^{\text {th }}$ pulse starting from the left hand side. The inverse pole figure reveals the sample microstructure and grain orientation. The different grains appear in different colours whereas grains with similar orientation are in similar colours. EBSD requires high quality surface preparation and minimum deformation of the sample surface. In Fig. 4b, the EBSD Image Quality (IQ) is superposed to the colour map.

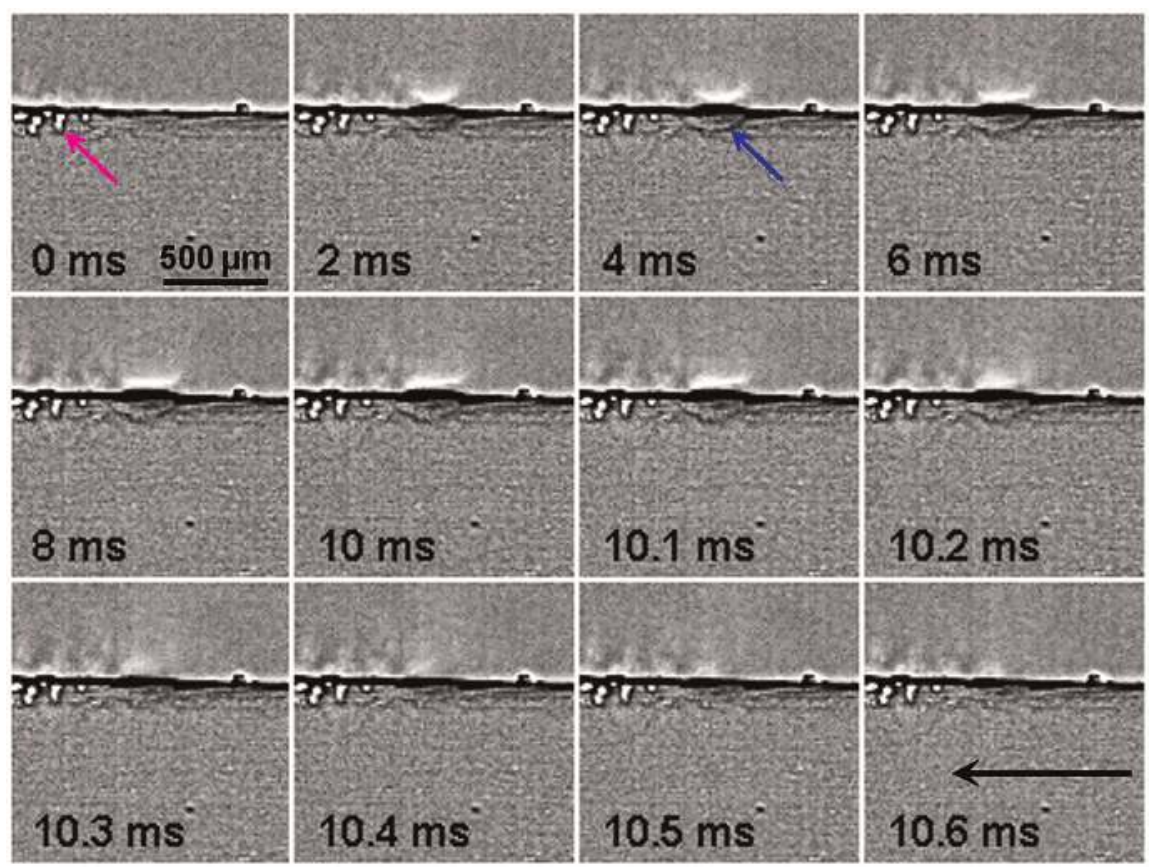

Fig. 3. X-ray radiograph of a single $10 \mathrm{~ms}$ pulse of $600 \mathrm{~W}$ in the conduction regime. Resolidification last approximately $0.5 \mathrm{~ms}$ after the end of the pulse. Pink arrow shows the porosity from previous pulses in keyhole regime. The blue arrow shows the fully formed melt pool. Black arrow shows the moving direction of the sample. Scale bar is indicated in the first frame and is the same for all.
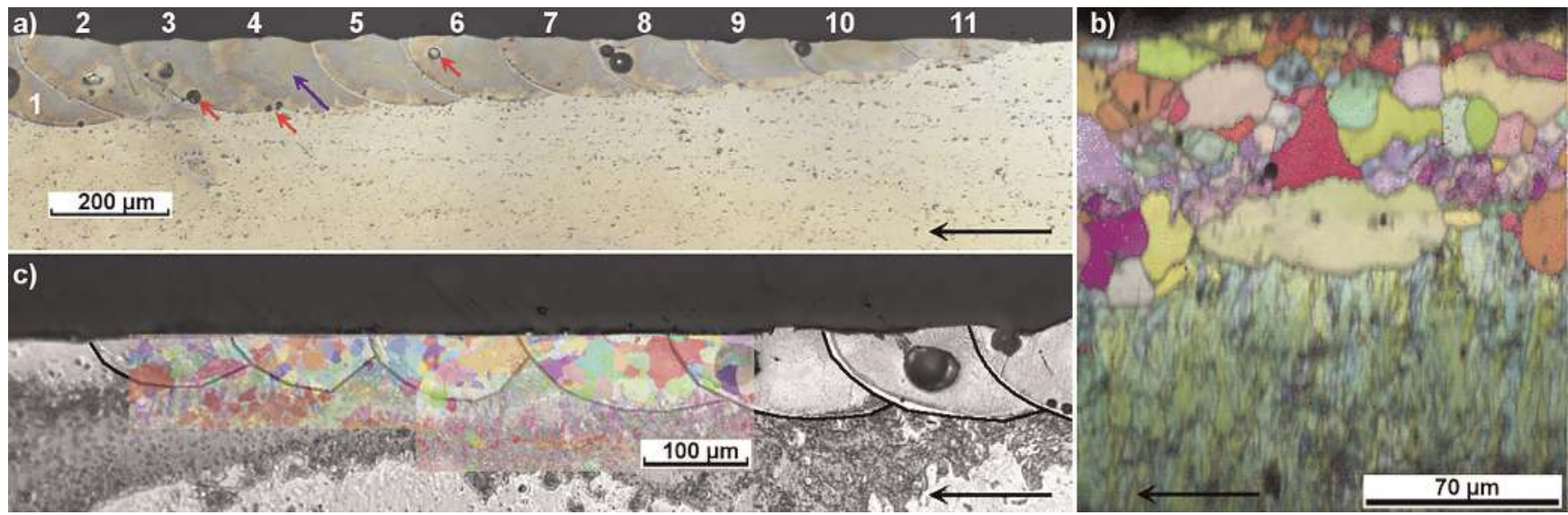

Fig. 4. (a) Micrograph of a series of pulses with increasing laser power from left to right. The different melt pools are revealed by etching with Weck's reagent. (b) EDSB map of one of the melt pool shown in (a). (c) Composite image of an optical micrograph and two EBSD maps to reveal the grains inside several melt pools with increasing laser power. 
The IQ is a measure of the quality of the EDSB pattern obtained for each point. It goes from black for no pattern or very bad pattern to fully transparent for perfect pattern. This has the advantage of revealing the grain boundaries, pores, cracks and regions of high deformation as they appear dark or black. The reason is that no good EBSD pattern is present in these regions. The melt pool microstructure is also very well separated from the bulk microstructure. In the re-solidified melt pool region, the microstructure consists of equiaxed grains with sizes of $15 \pm 1 \mu \mathrm{m}$. The bulk microstructure consists of deformed grains shown at the bottom of Fig. 3b with a low image quality.

Finally, Fig. 4c shows a composite image of the optical micrograph in black and white, with two EBSD maps superposed in colour. The contour of the melt pool was drawn in black to improve visibility. In contrast to Fig. $4 a$ and b, the melt pools shown in Fig. $4 \mathrm{c}$ are situated at the beginning of the experiment where the laser power was increased from left to right. The first melt pool on the left hand side is produced at approximately $150 \mathrm{~W}$. Laser powers below that value were not sufficient to melt the aluminium sample. The EBSD mapping was performed at lower magnification to get more than one melt pool per map and so can be easily superposed with the optical image. It can be seen that the grains are also equiaxed and well defined inside the melt pools. Outside the melt pools, the grains are not well defined and the image quality (not shown here) is very low. They appear as groups of small grains of similar colour separated by not measured area. This is additional proof of deformation of larger grains due to cold rolling of the aluminium piece.

The solidification of a keyhole is shown in Fig. 5a. For this experiment, the pulse power was increased to $1200 \mathrm{~W}$ for approximately $0.5 \mathrm{~ms}$ and then decreased by several steps in $12 \mathrm{~ms}$ to $60 \mathrm{~W}$, which was insufficient to maintain the melt pool. The power profile of the laser pulse is depicted in Fig. 5b. As can be seen, the size of the melt pool in the keyhole regime is much bigger and deeper than the one in conduction mode. Moreover, the aspect ratio (depth over diameter) is higher than 1 . The main characteristic of the keyhole (see red arrow, $5.5 \mathrm{~ms}$ ) is the region of metal vapour in the centre of the melt pool. The liquid melt pool

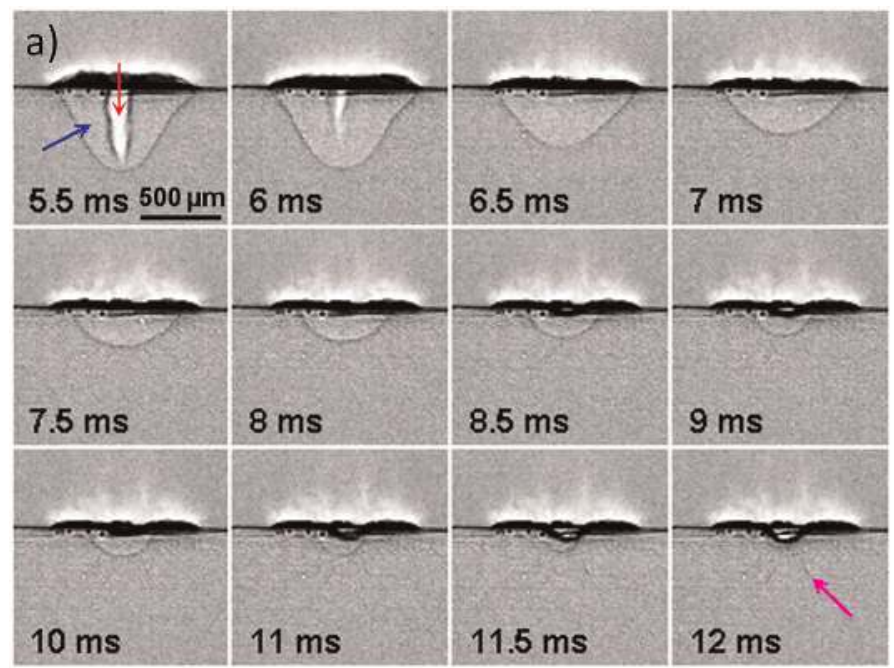

is also well visible around the keyhole due to its large size (see blue arrow, $5.5 \mathrm{~ms}$ ). The complete solidification time is much longer $(6 \mathrm{~ms})$ compared to the conduction for two reasons. Firstly, due to its larger size, almost 5 times deeper, it is expected that the melt pool takes longer to solidify. The second reason why the solidification is longer for the keyhole shown in Fig. 5a is due to pulse shaping shown in Fig. 5b. This pulse shaping strategy for the keyhole was selected to decrease the amount of cracks and hinder the pore formation observed in Fig. 2, in which the laser was kept constant at high power for the duration of the pulse. This has two mains consequences, first once the keyhole is formed the light absorption increases dramatically and this can lead to overheating of the keyhole and fluctuation of the keyhole with ultimately formation of pore at the bottom of the keyhole. Moreover, without pulse shaping, the drop in power at the end of the pulse provokes a very high cooling rate that can lead to the formation of large cracks as observed in Fig. 2. The influence of pulse shaping is visible in Fig. 5a on the frames from $8.5 \mathrm{~ms}$ to $10 \mathrm{~ms}$ where the size of the melt pool stays more or less constant during this time as the power is kept constant during this time (see Fig. 5b). The gradual decrease in power was meant to decrease the high temperature gradient observed during laser processing and so decrease the thermal stress induced during the process. Despite the pulse shaping, the thermal stresses are still high enough so that cracking is not completely hindered. Evidence of this is seen in Fig. 5a (11.5 and $12.0 \mathrm{~ms})$ where some small cracks are visible at the bottom right of the crater (see pink arrow, $12 \mathrm{~ms}$ ). But in contrast to Fig. 2, cracking is still much reduced and no porosity is formed.

A micrograph of the experiment in Fig. 5 is shown in Fig. 6a. The etching revealed the contour of the melt pool. Inside the melt pool, some solidification fronts are visible near the bottom of the keyhole and one is also visible near the top (red arrow). The last one (red arrow) is situated in the region where the melt pool was constant in the X-ray images $(t=8.5-10 \mathrm{~ms})$, therefore it is reasonable to think that they are correlated. The other solidification fronts near the bottom may come from fluctuations of the keyhole but no clear evidence is observable in the X-ray images.

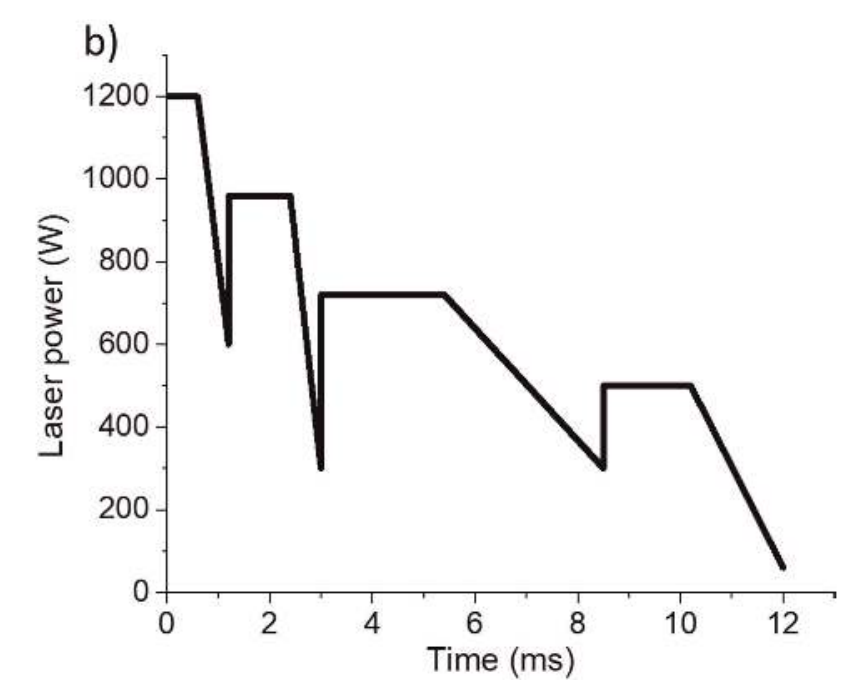

Fig. 5. (a) X-ray radiograph of the solidification of the keyhole welding experiment shown in Fig. 3. The solidification starts at approximately 6 ms after the end of the third plateau of power shown in (b) and lasts to $12 \mathrm{~ms}$ where the power reaches $60 \mathrm{~W}$. 
Figure $6 \mathrm{~b}$ is an EBSD map of the same region. In this figure, the grain orientation is shown by an inverse pole figure in colour and the IQ is shown in greyscale as in Fig. 4b. The IQ reveals well the grain boundaries and cracks in black. The material outside the melt pool appears very dark. This is due to a bulk microstructure heavily deformed which does not give good EBSD patterns. The IQ of the EBSD inside the re-solidified melt pool is good and the grains can be easily identified. Three different solidification regimes can be seen. At the bottom of the keyhole, where the solidification starts, the grains are small and equiaxed (outer equiaxed zone (OEZ)), with a similar size to what was observed for the melt pool in conduction regime (cf. Figure 4). This is followed by a region of columnar growth with elongated grains dominating the microstructure (columnar zone $(\mathrm{CZ})$ ). In this region, the grains have a length of around $41 \pm 5 \mu \mathrm{m}$ and a width of only $24 \pm 4 \mu \mathrm{m}$. Also, some intergranular cracks are visible in the optical image (Fig. 6a) and EBSD map (Fig. 6b). Finally close to the surface (inner equiaxed zone (IEZ)), the liquid was maintained by keeping an intermediate laser power, the microstructure is again composed of small equiaxed grains.

Figure $6 \mathrm{c}$ shows a superposition of the EBSD map on the optical image. The contour of the keyhole was drawn in white on the optical image for better visualisation. This confirms that the region of high IQ on the EBSD map corresponds with the re-solidified melt pool. However, the size of the melt pool appears slightly smaller on the EBSD map which is due to the re-polishing of the surface after etching the cross-section to create the EBSD map. Indeed, the first polishing was made to cut precisely in the centre of the keyhole and so really its maximum depth. After repolishing, the position is just slightly shifted off centre and, hence, shows a slightly smaller melt pool size. This re-polishing also explains why the cracks are not at the same position in the pictures.

By combining the X-ray imaging and metallographic observations, different mechanisms for microstructure formation can be related to different laser processing conditions. It is shown that at high cooling rates, such as the one obtained at the tip of the keyhole or during the cooling of a small melt pools in the conduction regime, the microstructure obtained is small equiaxed grains. This is illustrated in Fig. 7, where the interface velocity is plotted with time based on the X-ray images. In this figure, high velocity of the solidification interface is observed at the beginning of the keyhole solidification as well as during the complete solidification in conduction regime. Both cases correspond to regions with fine equiaxed microstructures. This is similar to the outer equiaxed zone observed in the casting process, where on the outside of the cast piece, a region of fine equiaxed grain is observed as the hot metal comes in contact with the cold mould [10]. In laser processing, the rapid cooling is achieved due to the small size of the melt pool and fast conduction cooling by the rest of the piece that stays at very low temperature [11]. For the keyhole experiment shown in Figs. 5 and 6, a drop in the re-solidification front's velocity is observed after $1 \mathrm{~ms}$. This is certainly due to the pulse shaping which helped the weld pool remain heated. Under these conditions and for interface velocity below $0.1 \mathrm{~m} \cdot \mathrm{s}^{-1}$, columnar growth is observed. This might be attributed to the fact that radiation energy is still deposited in the melt pool, leading to the re-melting of growing equiaxed crystals that are in front of the columnar interface. Therefore, the columnar growth is not inhibited and, hence, the columnar-to-equiaxed (CTE) transition is hindered in this case. Finally, when the laser is switch completely off during the keyhole experiment, the interface velocity increased again leading to a region of equiaxed growth. Although the interface velocity does not reach $0.1 \mathrm{~m} \cdot \mathrm{s}^{-1}$, the equiaxed microstructure cannot be explained by high cooling rate. The other explanation is that the continuous heat influx given by the laser during the pulse shaping has maintained the region surrounding the melt pool at high temperature. Hence the cooling from the solid is not as strong as at the start of the keyhole solidification. Moreover, the cooling from the top surface by convection may become more and more important. All these effects lead to a decrease of the thermal gradient inside the melt pool. A decrease of the thermal gradient is well known to favour the CTE transition and explains well the equiaxed microstructure observed [12]. The results presented in this article demonstrate that the final microstructure of laser processed material can be controlled by manipulating the heat input. This can be done by shaping the intensity profile of the laser beam, spatially and temporally, or by adjusting the trajectory of laser beam scanning. The latter has been recently reported for welding of aluminium using a wobbling laser beam [13].
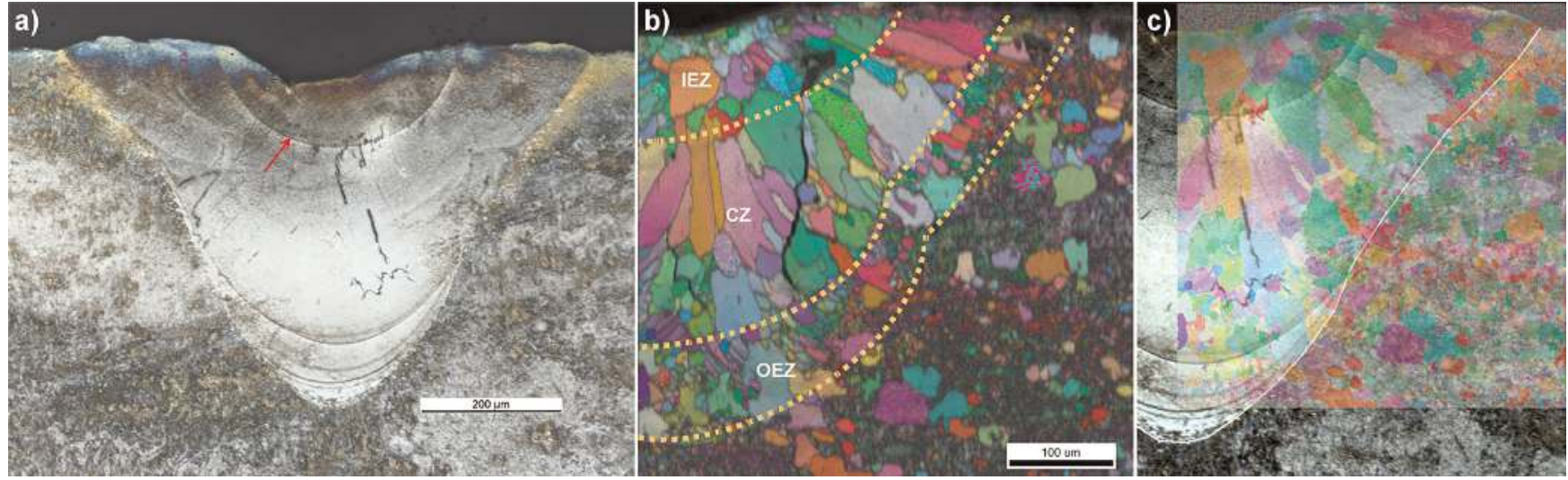

Fig. 6. a) Micrograph of the re-solidified melt pool created by a single pulse in the keyhole regime; b) EDSB map of the region in (a). Three regions are visible: the inner equiaxed zone (IEZ), the columnar zone (CZ) and outer equiaxed zone (OEZ); c) Superposition of image (a) and (b). 


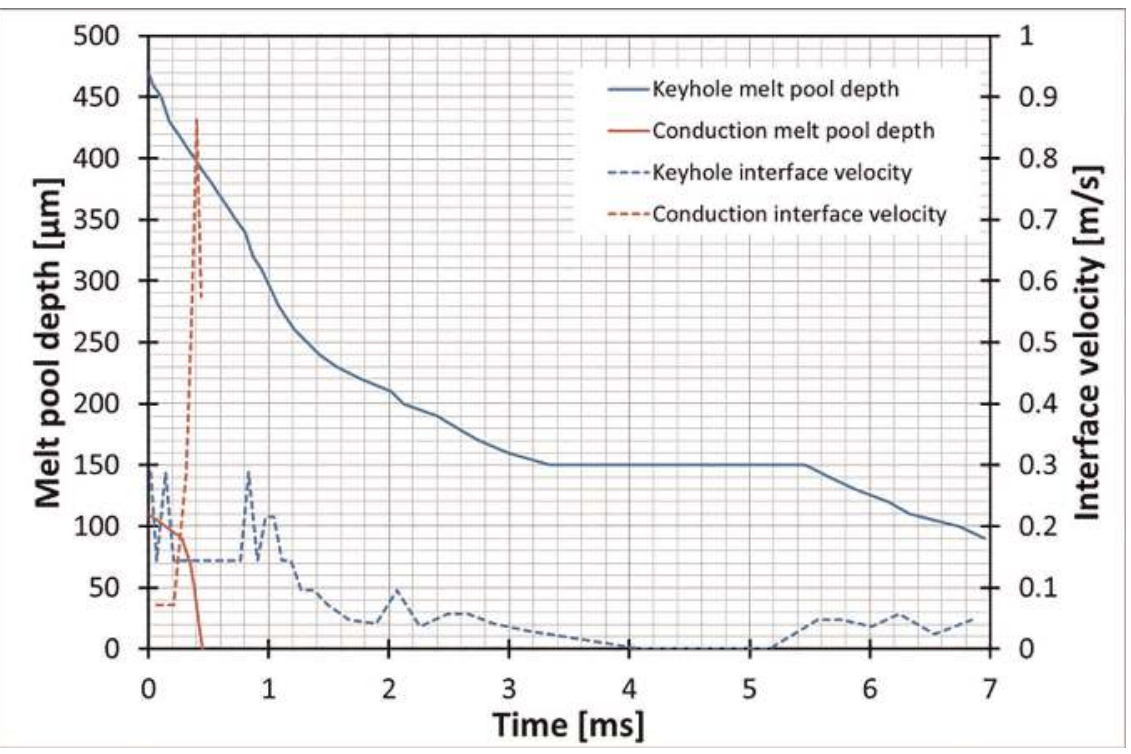

Fig. 7. Position and velocity of the interface as functions of time, as determined from the $\mathrm{X}$-ray images. The interface velocity is the discrete derivative of the position with time.

\section{Conclusions}

By combining in-situ real time X-ray radiography and post mortem metallographic observations, the microstructure formation for two cases, conduction and keyhole, was explained. A fine and equiaxed microstructure was observed in the conduction regime and at the beginning of the solidification of the keyhole. The equiaxed microstructure was related to a high interface velocity (above $0.1 \mathrm{~m} \cdot \mathrm{s}^{-1}$, see Fig. 6), which is the result of high cooling rates due to rapid cooling by the surrounding material similar to the outer equiaxed zone in casting. During keyhole solidification, after the initial equiaxed microstructure, a columnar microstructure is found. This is certainly due to the pulse shaping and maintaining of a hot melt pool by laser heating which prevents the growth of equiaxed crystals ahead of the columnar front. Finally at the centre of the keyhole, another region of equiaxed grains is observed and can be explained by a decrease of the thermal gradient inside the melt pool at the later stage of the cooling.

\section{References}

[1] S. Katayama, ed., Handbook of Laser Welding Technologies, Woodhead Publishing, 2013. DOI:10.1016/B978-0-85709-264-9.50025-8

[2] D.Y. You, X.D. Gao, S. Katayama: Sci. Technol. Weld. Join. 19 (2014) 181-201. DOI:10.1179/1362171813Y.0000000180

[3] T. Le-Quang, S.A. Shevchik, B. Meylan, F. Vakili Farahani, M. Olbinado, R. Alexander, K. Wasmer: Procedia CIRP. (2018). DOI:10.1016/j.procir.2018.08.055

[4] K. Wasmer, T. Le-Quang, B. Meylan, M. Olbinado, A. Rack, S.A. Shevchik: Procedia CIRP. (2018). DOI:10.1016/j.procir.2018.08.054

[5] M. Zhang, G. Chen, Y. Zhou, S. Li: Opt. Express. 21 (2013) 19997-20004. DOI:10.1364/OE.21.019997

[6] R. Fabbro: J. Phys. D. Appl. Phys. 43 (2010) 445501. DOI:10.1088/0022-3727/43/44/445501

[7] B.S. Yilbas, K. Danisman, Z. Yilbas : Meas. Sci. Technol. 2 (1991) 668. DOI:10.1088/0957-0233/2/7/016

[8] M. Courtois, M. Carin, P. Le Masson, S. Gaied, M. Balabane: J. Laser Appl. 26 (2014) 42001. DOI:10.2351/1.4886835

[9] R. Fabbro: J. Phys. D. Appl. Phys. 39 (2006) 394. DOI: $10.1088 / 0022-3727 / 39 / 2 / 023$
[10] C.-A. Gandin, M. Rappaz : Acta Metall. Mater. 42 (1994) 2233 2246. DOI:10.1016/0956-7151(94)90302-6

[11] C. Kenel, C. Leinenbach: J. Alloys Compd. 637 (2015) 242-247. DOI:10.1016/j.jallcom.2015.03.016

[12] J.D. Hunt: Mater. Sci. Eng. 65 (1984) 75-83. DOI:10.1016/0025-5416(84)90201-5

[13] C. Hagenlocher, M. Sommer, F. Fetzer, R. Weber, T. Graf: Mater. Des. 160 (2018) 1178-1185. DOI:10.1016/j.matdes.2018.11.009

(Received December 15, 2018; accepted June 27, 2019; online since November 8, 2019)

\section{Correspondence address}

Dr. Kilian Wasmer

Laboratory for Advanced Materials Processing (LAMP)

Swiss Federal Laboratories for Materials Science and Technology (Empa)

Feuerwerkerstrasse 39

3603 Thun

Switzerland

Tel.: +41587656271

Fax: +41587656990

E-mail: kilian.wasmer@empa.ch

Web: https://www.empa.ch/web/s204/kilian-wasmer

\section{Bibliography}

DOI 10.3139/146.111838

Int. J. Mater. Res. (formerly Z. Metallkd.)

111 (2020) 1; page 17-22

(C) Carl Hanser Verlag GmbH \& Co. KG

ISSN 1862-5282 